\title{
Tissue Characterization of Coronary Plaques and Assessment of Thickness of Fibrous Cap Using Integrated Backscatter Intravascular Ultrasound - Comparison With Histology and Optical Coherence Tomography -
}

Masanori Kawasaki, MD, PhD; Arihiro Hattori, MD; Yoshiyuki Ishihara, MD; Munenori Okubo, MD, PhD; Kazuhiko Nishigaki, MD, PhD; Genzou Takemura, MD, PhD; Masanao Saio, MD, PhD; Tsuyoshi Takami, MD, PhD; Shinya Minatoguchi, MD, PhD

\begin{abstract}
Background: The purpose of this study was to develop a new online integrated backscatter intravascular ultrasound (IB-IVUS) system and to validate its ability to measure fibrous cap thickness by comparing IB-IVUS images with those from optical coherence tomography (OCT).

Methods and Results: Images were acquired from 125 segments of 26 coronary arteries obtained at autopsy from 11 cadavers. In the training study $(n=30), 242$ regions-of-interest on color-coded maps were compared with histology. In the validation study, 95 cross-sections were diagnosed by IB-IVUS and histology. In 24 patients with stable angina, 28 arterial cross-sections were imaged by IB-IVUS and OCT in vivo. In the training study, cutoff values of -39 decibels $(\mathrm{dB})$ and $-17 \mathrm{~dB}$ were the optimal predictors of lipid pool/fibrosis and fibrosis/calcification, respectively, with $38-\mathrm{MHz}$ mode; $-42 \mathrm{~dB}$ and $-20 \mathrm{~dB}$, respectively, with $43-\mathrm{MHz}$ mode. In the validation study, IB classified the fibrous, lipid-rich and fibrocalcific components with an accuracy of $92 \%, 91 \%$ and $95 \%$, respectively. Agreement between the histological and IB-IVUS diagnoses was excellent (Cohen's $\kappa=0.83$ ). There was a correlation between the fibrous cap thickness measured by IB-IVUS and OCT $(r=0.74, P<0.001)$.
\end{abstract}

Conclusions: The IB-IVUS system with improved resolution provides high diagnostic accuracy for the analysis of the tissue characteristics of coronary plaques, and enables estimation of the thickness of the fibrous cap in the clinical setting. (Circ J 2010; 74: 2641-2648)

Key Words: Coronary plaque; Fibrous cap; Integrated backscatter; Intravascular ultrasound; Optical coherence tomography

A pproximately 30 years ago, Horie et al demonstrated in a pathological study that plaque rupture into the vessel lumen may precede, and cause, thrombus formation and the onset of acute myocardial infarction. ${ }^{1}$ Later, Mizuno et al demonstrated in an angioscopic study that disruption or erosion of vulnerable plaques and subsequent thromboses are the most frequent cause of acute coronary syndrome (ACS). ${ }^{2}$ The stability of atherosclerotic plaque is related to its histological composition and the thickness of the fibrous cap. Therefore, tissue characterization of coronary plaques is important for understanding and preventing ACS. Accurate in vivo identification of the tissue characteristics of coronary plaques may enable vulnerable plaques to be identified before the development of ACS.

\section{Editorial p 2554}

Intravascular optical coherence tomography (OCT) can provide high-resolution, cross-sectional images of plaques in situ, with an axial resolution of $10 \mu \mathrm{m}$ and a lateral resolution of $20 \mu \mathrm{m} .{ }^{3,4}$ Furthermore, OCT images of human coronary atherosclerotic plaques obtained in vivo have provided detailed structural information near the surface of the coronary lumen. ${ }^{5-9}$ The thickness of the fibrous cap can be measured with OCT, and a good correlation between OCT and histological examination has been reported. ${ }^{10}$

In addition to OCT, intravascular ultrasound (IVUS) has been used to image coronary plaques. We developed inte-

Received June 8, 2010; revised manuscript received July 15, 2010; accepted July 27, 2010; released online October 9, 2010 Time for primary review: 22 days

Regeneration \& Advanced Medical Science (M.K., A.H., Y.I., M.O., K.N., G.T., S.M.), Department of Immunopathology (M.S., T.T.), Gifu University Graduate School of Medicine, Gifu, Japan

Mailing address: Masanori Kawasaki, MD, PhD, Regeneration \& Advanced Medical Science, Gifu University Graduate School of Medicine, 1-1 Yanagido, Gifu 501-1194, Japan. E-mail: masanori@ya2.so-net.ne.jp

ISSN-1346-9843 doi:10.1253/circj.CJ-10-0547

All rights are reserved to the Japanese Circulation Society. For permissions, please e-mail: cj@j-circ.or.jp 


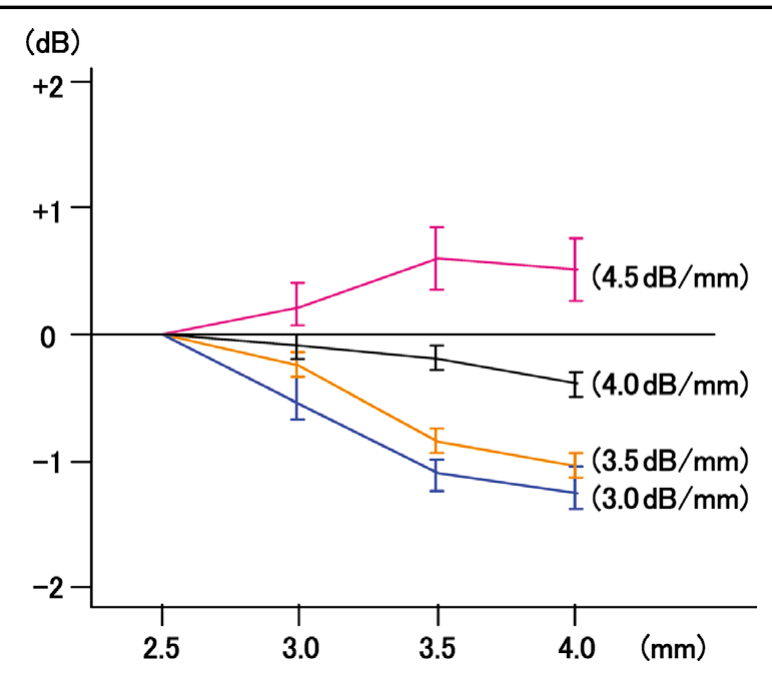

Figure 1. With the inclusion of attenuation by flowing blood, an overall attenuation of $4.0 \mathrm{~dB} / \mathrm{mm}$ was determined to be the most appropriate, and this value was used to correct for ultrasound signal attenuation. $\mathrm{dB}$, decibels.

grated backscatter (IB)-IVUS, which enabled characterization of the tissue components of human coronary arterial plaques both ex vivo and in the clinical setting. ${ }^{11-15}$ We recently improved the lateral resolution of IB-IVUS images by increasing both the vector lines of the radiofrequency signal and the digital sampling rate. However, the ability of this new system to characterize coronary plaques and to measure the thickness of the fibrous cap has not been evaluated.

Therefore, the purpose of the present study was to evaluate our new IB-IVUS system for tissue characterization of coronary plaque and to validate its ability to measure the thickness of fibrous caps by comparing IB-IVUS images with OCT images.

\section{Methods}

\section{IB-IVUS Equipment and Data Acquisition}

A personal computer (Windows XP Professional, CPU: $3.4 \mathrm{GHz}$ ) equipped with newly developed custom software was connected to an IVUS imaging system (VISIWAVE, Terumo, Japan) to obtain the radiofrequency signal, signal trigger and video image outputs. With the new system, we set 512 vector lines of ultrasound signal around the circumference, compared with the 256 vector lines used in the previous system. In addition, the sampling rate of ultrasound was improved from $100 \mathrm{MHz}$ to 320 and $400 \mathrm{MHz}$. IB values for each tissue component were calculated using a fast Fourier transform, and expressed as the average power, measured in decibels $(\mathrm{dB})$, of the frequency component of the backscattered signal from a small volume of tissue. Ultrasound backscattered signals were acquired using a $38-$ and $43-\mathrm{MHz}$ mechanically-rotating IVUS catheter (ViewIT, Terumo, Tokyo, Japan), digitized and subjected to spectral analysis. We used the 43-MHz ultrasound mode to improve the image quality because the penetration depth of the ultrasound signal is limited in the coronary arteries with ultrasound frequencies greater than $43 \mathrm{MHz}$. We also used the $38-\mathrm{MHz}$ ultrasound mode, which is similar to the $40 \mathrm{MHz}$ catheter used in our previous study, but has a relatively greater penetration depth than the $40-\mathrm{MHz}$ mode. The tissue IB values were calibrated by subtracting the IB values from the IB value of a stainless steel needle placed at a distance of $1.5 \mathrm{~mm}$ from the catheter. IB-IVUS color-coded maps were constructed from the IB values by use of customized software. Conventional IVUS images and IB-IVUS color-coded maps were immediately displayed side-by-side on a monitor. Color-coded maps of the entire coronary arteries were finally constructed after excluding the vessel lumen and area outside the external elastic membrane by manually tracing these components on the conventional IVUS images. With a transducer frequency of 38 or $43 \mathrm{MHz}$, the wavelength was calculated as 36 or $41 \mu \mathrm{m}$, respectively, assuming a tissue sound speed of approximately $1,560 \mathrm{~m} / \mathrm{s}$.

\section{Histological Analysis}

Images were acquired from 125 human coronary segments (testing set: 30 cross-sections; validation set: 95 cross-sections) of 26 coronary arteries obtained at autopsy from 11 cadavers, of which 7 had a history of symptomatic cardiovascular disease (64\%). The coronary arteries were dissected within $24 \mathrm{~h}$ of death, immediately fixed in $10 \%$ buffered formalin solution and stored for more than $24 \mathrm{~h}$. Ring-like arterial sites obtained at the same level as the imaging study were decalcified for $5 \mathrm{~h}$, embedded in paraffin and cut perpendicular to the longitudinal axis of the artery into $2-\mu \mathrm{m}$ transverse sections. They were stained with hematoxylineosin, elastic van Gieson and Masson's trichrome. In the training study, 3 pathologic subsets were identified in each region of interest (ROI): (1) lipid pool (extracellular lipid, macrophage, micro-calcification and/or form cells), (2) fibrosis and (3) calcification. Necrotic core that consisted of lipid pool, micro-calcification and remnants of foam cells and/or dead lymphocytes were expressed as "lipid pool" with the IB-IVUS system. In the validation study, coronary arterial cross-sections were classified into 3 categories: (1) fibrocalcific, (2) fibrous and (3) lipid-rich according to our previous study. ${ }^{13}$ IB-IVUS images were validated with the corresponding histology to determine diagnostic accuracy. Histological classification of coronary segments was performed by the consensus of 2 pathologists who were unaware of the IBIVUS images. The experimental protocol was approved by the institutional ethics committee. Informed consent was given by the relatives of the patients for the ex vivo study in all cases.

\section{Correlation Between IB-IVUS Images and Histology}

Arterial segments were subjected to IB-IVUS imaging in saline at a temperature of $37^{\circ} \mathrm{C}$. Each IB-IVUS/histology pair was randomly assigned to the training set $(n=30)$ or validation set $(n=95)$. In the training study, small $(0.3 \times 0.3 \mathrm{~mm})$ ROIs $(\mathrm{n}=242)$ located $1.5 \pm 0.1 \mathrm{~mm}$ from the center of the catheter were selected from the IB-IVUS images, based on comparison with histology. This size of ROI was used in our previous study that compared histology and IB-IVUS images. ${ }^{13}$ IB values for calcification $(n=41)$, fibrosis $(n=102)$ and lipid pool $(n=99)$ from each ROI were calculated and subjected to statistical analysis. It has been reported that the attenuation of flowing blood with a $40-\mathrm{MHz}$ of ultrasound signal is $0.8 \mathrm{~dB} / \mathrm{mm} .{ }^{16}$ To evaluate overall ultrasound signal attenuation, we initially measured IB values of the same lesions $(n=$ $10)$, moving the lesions $2.5-4.0 \mathrm{~mm}$ from the IVUS catheter. Including the attenuation by flowing blood, an overall attenuation of $4.0 \mathrm{~dB} / \mathrm{mm}$ was determined to be the most appropriate, and this value was used to correct for ultrasound signal attenuation (Figure 1). Therefore, when the color-coded maps were constructed, we corrected each IB value by adding 


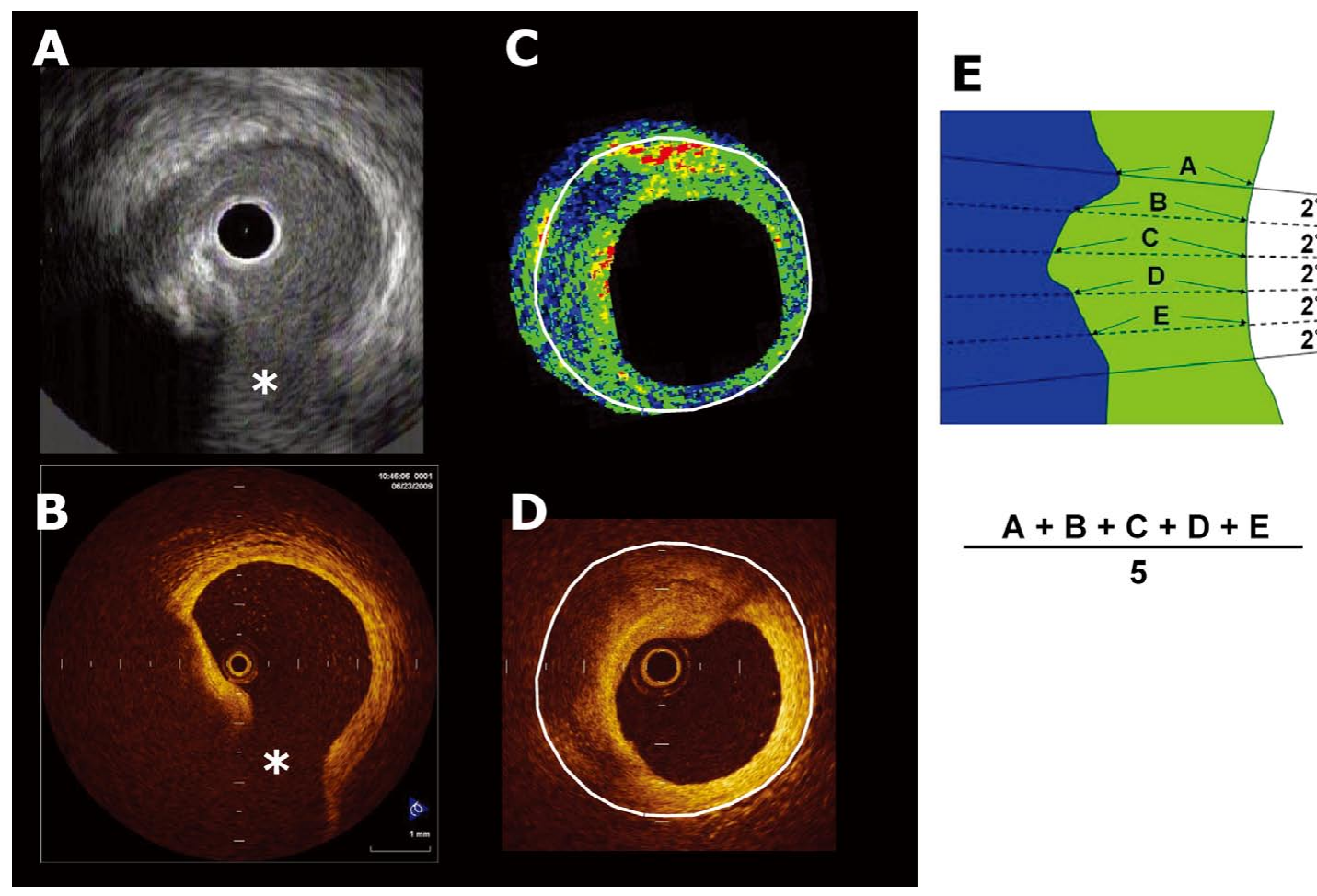

Figure 2. Selection of the same coronary segments to image using the distance from easily-definable side branches and calcification as reference markers to ensure that integrated backscatter intravascular ultrasound (IB-IVUS) and optical coherence tomography (OCT) images were compared at the same site. (A) Conventional IVUS image. (B) Corresponding OCT image. (C) IB-IVUS image. (D) Corresponding OCT image. (E) Fibrous caps that overlaid lipid pool were divided into regions-of-interest (ROIs) (every $10^{\circ}$ from the center of the vessel) and the thickness of the fibrous caps was measured as an average. The average thickness of the fibrous cap was measured by averaging the thickness of the fibrous cap every $2^{\circ}$ within the ROls. ${ }^{*}$ Septal branch.

$4.0 \mathrm{~dB} / \mathrm{mm}$ when the ROI was located $1.5 \mathrm{~mm}$ further from the catheter and subtracting $4.0 \mathrm{~dB} / \mathrm{mm}$ when the ROI was located $1.5 \mathrm{~mm}$ closer to the catheter. We previously confirmed that formalin fixation does not significantly affect the quantitative echo character of plaque tissue of human aortic walls, and that the IB values of arteries with a constant pressure before fixation are similar to those at the same site of non-pressured arteries after fixation. ${ }^{13}$ To clarify the rotational and cross-sectional position of the included segment, multiple surgical needles were carefully inserted into the coronary arteries before IB-IVUS imaging to serve as reference points in the comparison of the IB-IVUS images with histology. This method has been successfully used in previous studies that compared histology and IVUS images. ${ }^{13,17,18}$ After placing the needles, the catheter was advanced into the coronary arteries and pulled back at $0.5 \mathrm{~mm} / \mathrm{s}$ by activating an autopullback device. IB-IVUS images were acquired at the interval of 1 image per second during the auto-pullback procedure. Cross-sectional images from sites containing surgical needles and from sites within $1 \mathrm{~mm}$ of the needles were obtained to ensure that IB-IVUS and histology were compared at the same site. After the images were acquired, imaging sites were sutured to serve as reference points to compare IVUS images with histology.

Color-coded maps consist of 4 major components (fibrous [green], dense fibrosis [yellow], lipid pool [blue], and calcification [red]). Based on the successful application in our previous study, ${ }^{13}$ in the present study, IB-IVUS lesions that included lipid pool [blue] occupying $>30 \%$ of the entire crosssection were diagnosed as lipid-rich and lesions that included calcification [red] occupying $>1 \%$ of the entire cross-section were diagnosed as fibrocalcific, because we could recognize a calcified mass in the corresponding histological images when the red area was $>1 \%$ on the IB-IVUS images. Otherwise, the lesions were diagnosed as fibrous.

\section{Comparison of the Thickness of the Fibrous Cap Measured by IB-IVUS and OCT In Vivo}

Patients were excluded if they had had unstable angina or a myocardial infarction within the previous 3 months or had an ejection fraction $\leq 30 \%$. In 42 consecutive patients undergoing routine selective percutaneous coronary intervention, a total of 28 arterial cross-sections that consisted of lipid overlaid by a fibrous cap were imaged by both IVUS and OCT in 24 patients with stable angina pectoris. Informed consent was given by all patients. To prevent coronary spasm, we administered an optimal intracoronary dose of isosorbide dinitrate before the measurements. A 0.016-inch OCT catheter (Imagewire, LightLab Imaging, Westford, MA, USA) was advanced into the coronary arteries. IB-IVUS and OCT (M2 OCT Imaging system, LightLab Imaging) were performed in each patient at the same site without significant stenosis as described next.

IB-IVUS images were obtained every second using an 


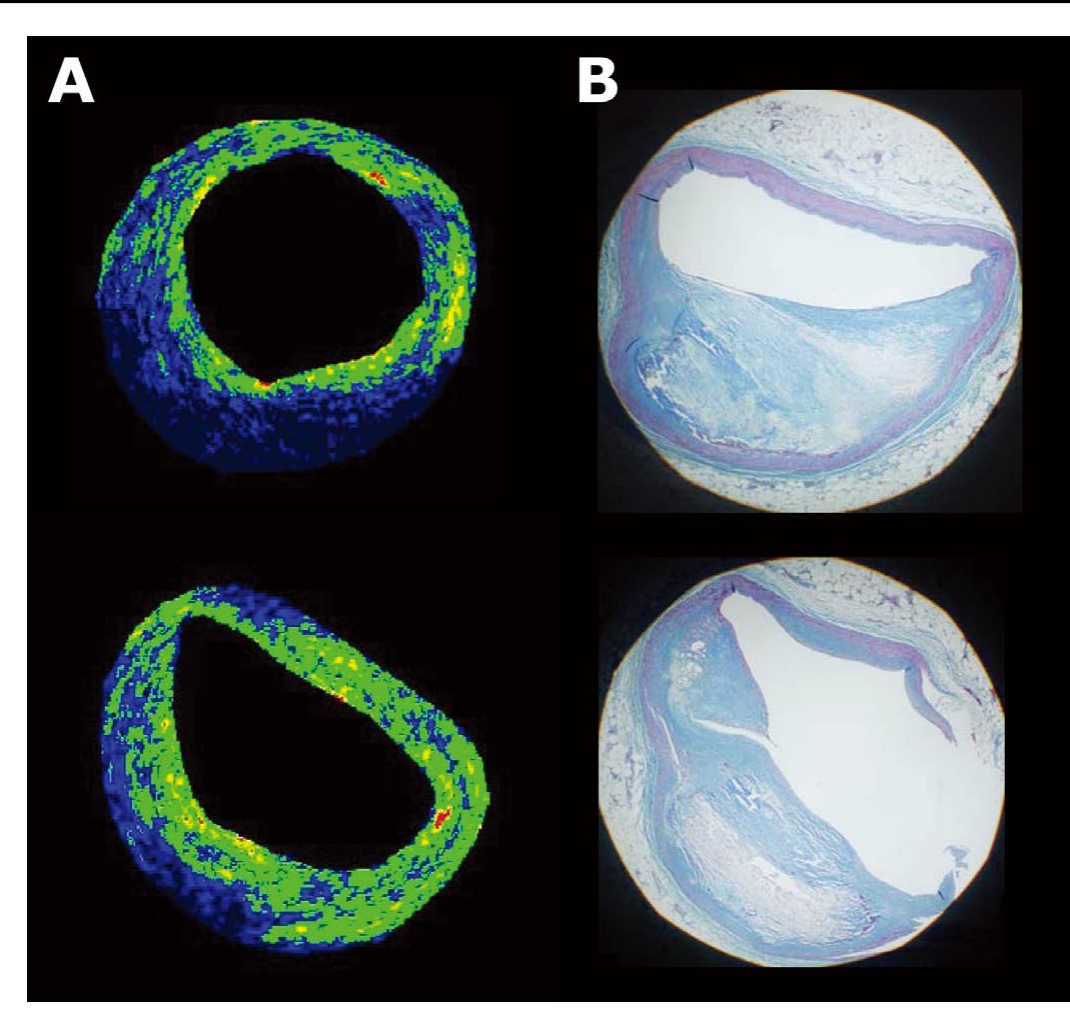

Figure 3. (A) Representative images obtained by integrated backscatter intravascular ultrasound and the corresponding histological images (B).

automatic pullback device at a rate of $0.5 \mathrm{~mm} / \mathrm{s}$. OCT images were obtained using an automatic pullback system at a rate of $0.5 \mathrm{~mm} / \mathrm{s}$. IB-IVUS imaging construction was possible every $0.5 \mathrm{~mm}$, whereas OCT imaging construction was possible every $0.03 \mathrm{~mm}$. Therefore, we selected the IB-IVUS images first to compare with the OCT images. We then identified the same coronary segments in the OCT images using the distance from easily-definable side branches and calcification as a reference marker to ensure that IB-IVUS and OCT were compared at the same site. The cross-sections that did not have sufficient image quality to analyze tissue characterization were excluded from the comparison. In the IB-IVUS analysis, IB-IVUS images were processed by a smoothing method that averaged 9 IB values in 9 pixels located in a square field of the color-coded maps in order to reduce uneven surfaces of the tissue components produced by the signal noise. Fibrous caps that overlaid lipid pool were divided into ROIs (every $10^{\circ}$ rotation from the center of the vessel lumen) and the average thickness was determined by averaging the thickness of fibrous cap every $2^{\circ}$ within the ROIs (Figure 2). The areas where the radial axis from the center of the vessel lumen crossed the tangential line of the vessel surface at less than $80^{\circ}$ were excluded from the comparison.

\section{Statistical Analysis}

Values are reported as the mean \pm SD. The significance of the differences of IB values among the tissue characteristics of coronary plaques were tested using analysis of variance followed by Fisher's least significant difference as the posthoc test. Analysis of receiver-operating characteristic curves was performed to determine the cutoff points for differentiating each tissue component. For estimation of the predictive accuracy of the IB values at the selected cutoff points, the sensitivity, specificity, positive predictive value and negative predictive value were calculated. The agreement between histological classification and IB-IVUS classification was quantified by Cohen's $\kappa$ test of concordance. ${ }^{19} \mathrm{~A} \kappa$ value of $0.61-0.80$ indicates good agreement, and 0.81-1.0 indicates excellent agreement. ${ }^{20}$ The correlation between the thicknesses of the fibrous cap measured by IB-IVUS and OCT was determined by linear regression analysis. Statistical analyses were performed using Stat View version 5.0 (SAS Institute, Cary, NC, USA).

\section{Results}

\section{Reproducibility and Reliability of Data}

We determined the inter-catheter variability of cross-sectional areas in 18 recordings that were measured by 1 observer using 6 catheters (ViewIT, Terumo, Japan) selected at random. The inter-catheter variability of the lipid pool cross-sectional area and the fibrous cross-sectional area was $6.0 \pm 6.5 \%$ and $3.0 \pm$ $3.0 \%$, respectively.

We used a stainless steel tube with a known diameter as the standard and measured its diameter by IVUS to ensure the accuracy of thickness measurements by the new system. Using stainless steel tubes with diameters of 2.00, 3.00 and $4.00 \mathrm{~mm}$, and assuming the speed of sound in saline was $1,530 \mathrm{~m} / \mathrm{s}$, the diameters measured by the IVUS catheter were $2.00 \pm 0.02,3.03 \pm 0.01$ and $4.06 \pm 0.01 \mathrm{~mm}$, respectively.

\section{Comparison of IB Values and Histology Ex Vivo}

In the training study, a total of 242 ROIs $(0.3 \times 0.3 \mathrm{~mm})$ with typical histology were examined in 30 arterial segments to compare the IB values and the histological diagnosis. The typical tissue components comprising calcification $(n=41)$, fibrosis $(n=102)$ and lipid pool $(n=99)$ of these ROIs were 
selected. With the 38-MHz ultrasound mode, the average IB values for these tissue components in each ROI were $-7.3 \pm$ $6.5,-27.0 \pm 5.3$ and $-51.2 \pm 3.3 \mathrm{~dB}$, respectively; however, with the $43-\mathrm{MHz}$ ultrasound mode, the average IB values were $-10.6 \pm 6.1,-30.4 \pm 4.9$ and $-54.0 \pm 3.9 \mathrm{~dB}$, respectively. The differences among the IB values for lipid pool, fibrosis and calcification were significant $(\mathrm{P}<0.001)$. IB values were highest for calcification and lowest for lipid pool. There was no overlap between the IB values for lipid pool and calcification. According to our analysis of the receiver-operating characteristic curves, an IB value $\leq-39 \mathrm{~dB}$ (area under curve [AUC] $=0.98$ ) was the most reliable cutoff point for discriminating lipid pool (90\% sensitivity, $92 \%$ specificity) and

\begin{tabular}{|lrrrr|}
\hline \multirow{4}{*}{$\begin{array}{l}\text { Table 1. Overall Agreement Between IB-IVUS and } \\
\text { Histological Diagnosis (Validation Study) } \\
\end{array}$} & \multicolumn{4}{c|}{ Histological diagnosis } \\
\cline { 2 - 5 } & LR & FL & FC & Total \\
IB-IVUS diagnosis & & & & \\
LR & 30 & 3 & 2 & 35 \\
FL & 3 & 29 & 1 & 33 \\
FC & 1 & 1 & 25 & 27 \\
Total & 34 & 33 & 28 & 95 \\
\hline
\end{tabular}

Cohen's $\kappa=0.83$ (95\%Cl 0.73-0.92).

IB-IVUS, integrated backscatter intravascular ultrasound; LR, lipidrich; FL, fibrous; FC, fibrocalcific; $\mathrm{Cl}$, confidence intervals.

\begin{tabular}{|lccccc|}
\hline \multicolumn{5}{|c|}{ Table 2. Diagnostic Accuracy of IB-IVUS for Plaque Characterization (Validation Study) } & \\
& Sensitivity & Specificity & PPV & NPV & Accuracy \\
LR (35 segments) & $88(77-99)$ & $92(83-100)$ & $86(75-97)$ & $93(85-100)$ & $91(81-100)$ \\
FL (33 segments) & $88(77-99)$ & $94(86-100)$ & $88(77-99)$ & $94(86-100)$ & $92(83-100)$ \\
FC (27 segments) & $97(91-100)$ & $89(77-100)$ & $96(89-100)$ & $93(83-100)$ & $95(87-100)$ \\
\hline
\end{tabular}

Data are percentages. Numbers in parentheses are $95 \% \mathrm{Cl}$.

PPV, positive predictive value; NPV, negative predictive value. Other abbreviations see in Table 1.

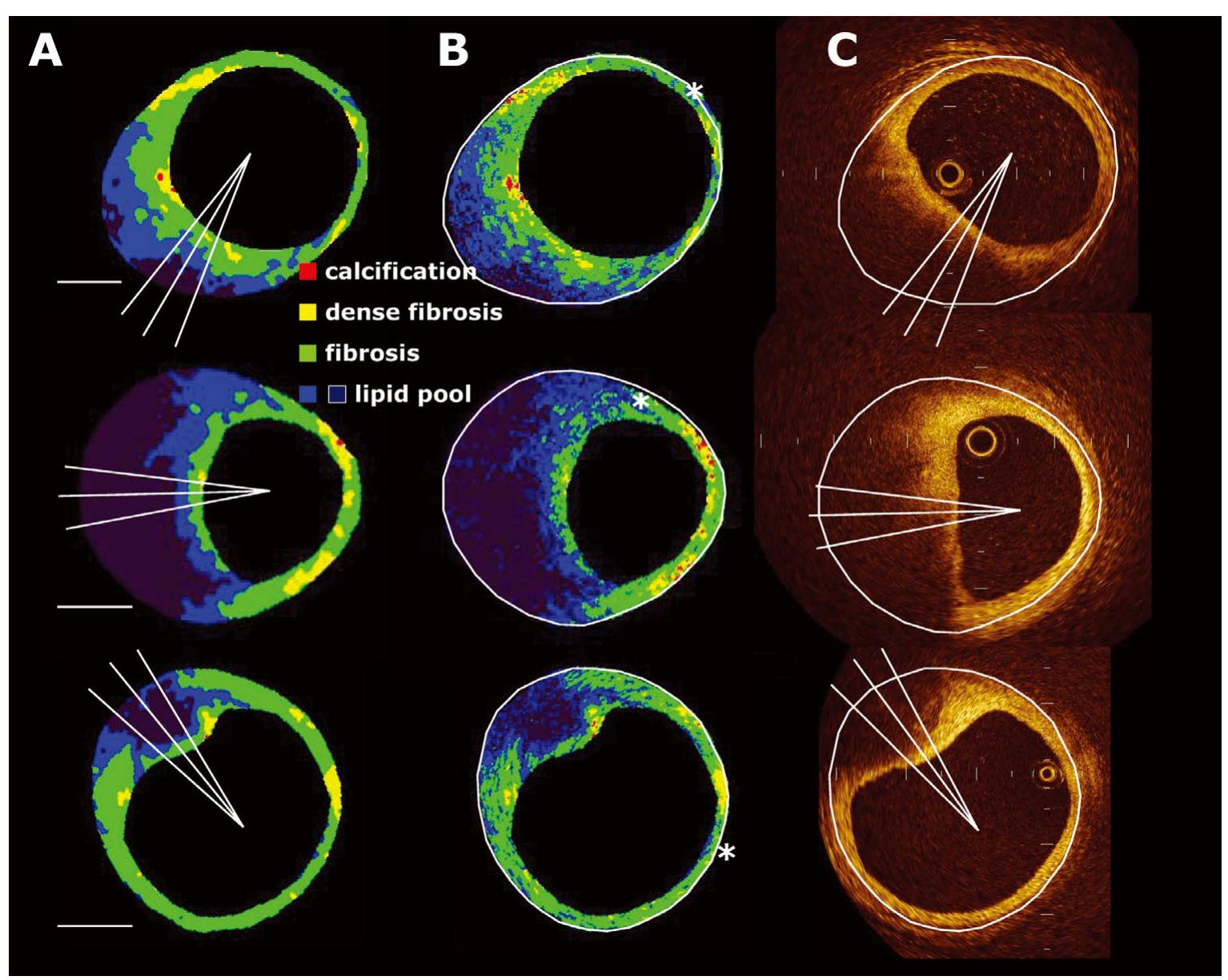

Figure 4. (A) Representative integrated backscatter intravascular ultrasound (IB-IVUS) images processed by a smoothing method. (B) Original IB-IVUS images and corresponding optical coherence tomography images (C). *Attenuation by guidewire. Bar $=1 \mathrm{~mm}$. 

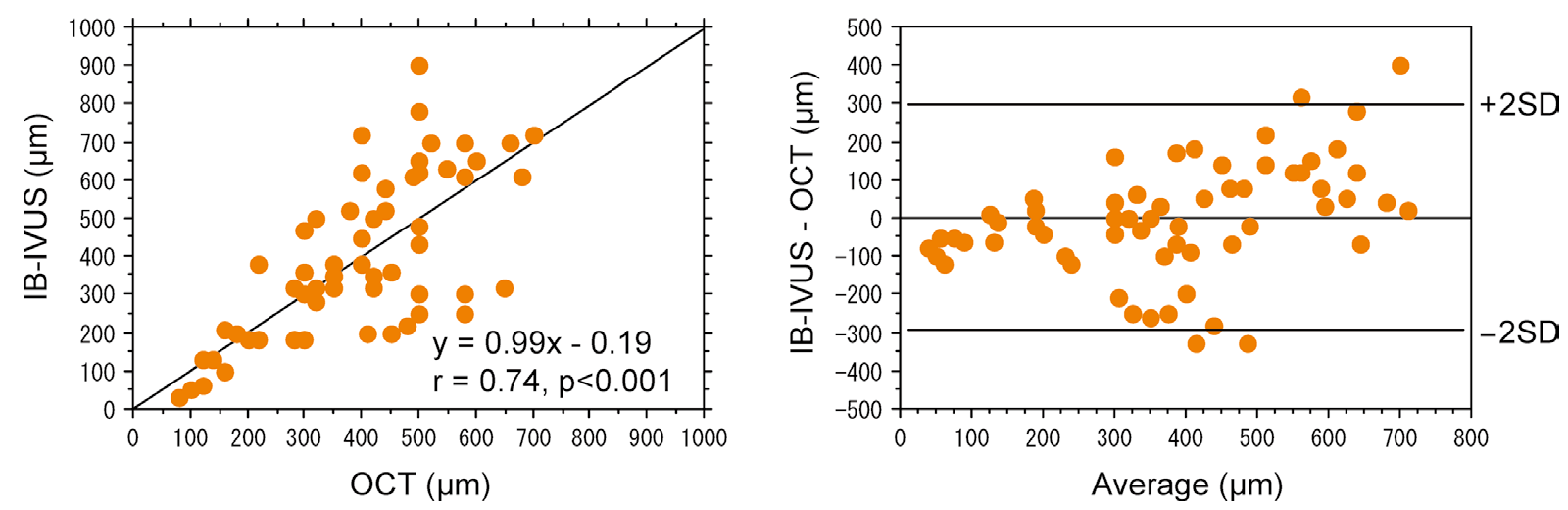

Figure 5. (Left) Correlation between the thickness of the fibrous cap measured by integrated backscatter intravascular ultrasound (IB-IVUS) and optical coherence tomography (OCT). (Right) Bland-Altman plot.

fibrosis (94\% sensitivity, 93\% specificity), and an IB value $>-17 \mathrm{~dB}(\mathrm{AUC}=0.99)$ was the most reliable cutoff point for discriminating calcification and fibrosis with the $38-\mathrm{MHz}$ mode. An IB value $\leq-42 \mathrm{~dB}$ (AUC $=0.98$ ) was the most reliable cutoff point for discriminating lipid pool and fibrosis, and an IB value $>-20 \mathrm{~dB}(\mathrm{AUC}=0.99)$ was the most reliable cutoff point for discriminating calcification and fibrosis with the 43-MHz mode.

\section{Diagnostic Accuracy of Classifying Arterial Plaque}

Based on these cutoff points, 2-dimensional color-coded maps of the tissue characteristics were constructed. We also compared the IB-IVUS and histological diagnoses based on arterial cross-sections. A total of 95 cross-sections were diagnosed as fibrocalcific, fibrous or lipid-rich by the IB-IVUS reader who was unaware of the histological diagnoses. There was no difference in the diagnosis of the images obtained using either the 38 - or $43-\mathrm{MHz}$ ultrasound signal. The overall agreement between the classifications made by IB-IVUS and histology (lipid-rich: $n=35$, fibrous: $n=33$ and fibrocalcific: $\mathrm{n}=27)$ was excellent $(\kappa=0.83,95 \%$ confidence intervals 0.73-0.92) (Figure 3, Table 1). Table 2 shows the sensitivity, specificity, positive and negative predictive values and accuracy for classifying the tissue components as lipid-rich, fibrous or fibrocalcific.

\section{Thickness of Fibrous Cap Measured by IB-IVUS and OCT}

As shown in Figures $\mathbf{4}$ and $\mathbf{5}$, the thickness of the fibrous cap measured by IB-IVUS significantly correlated with that measured by OCT $(y=0.99 x-0.19, r=0.74, P<0.001)$. In the Bland-Altman plot, the mean difference between the thickness of fibrous cap measured by IB-IVUS and OCT (IB-IVUSOCT) was $-2 \pm 147 \mu \mathrm{m}$.

\section{Discussion}

In the present study, we demonstrated that tissue characterization with an IB-IVUS system with improved resolution and smoothing method was feasible and reliable. The thickness of the fibrous cap measured by IB-IVUS in vivo correlated with that measured by OCT. That finding suggests the possibility of estimating the thickness of the fibrous cap in the clinical setting using this improved IB-IVUS, although we did not believe it was appropriate to perform rigorous estimation of the fibrous cap thickness in the present study.

\section{Diagnostic Accuracy of Tissue Characterization}

A previous study demonstrated that the tissue components of coronary plaques could be characterized using IVUS and an autoregressive classification scheme rather than depending on the classic Fourier method. ${ }^{21}$ In that study, parameters such as the frequencies at maximum and minimum power and the slope of the regression line of ultrasound backscattered signals were taken into account in the analysis. The autoregressive method classified fibrous, fibrofatty, necrotic core and dense calcium with a high predictive accuracy of $87 \%$, $87 \%, 88 \%$ and $97 \%$, respectively. However, an analysis using IB values classified fibrous, lipid-rich, and fibrocalcific plaque components with a predictive accuracy of $93 \%, 90 \%$ and $96 \%$, respectively. The values obtained using IB-IVUS were similar to those obtained using the autoregressive classification scheme. In the present study, the IB cutoff points for differentiating between lipid pool and fibrosis, and fibrosis and calcification were determined by analysis of receiveroperating characteristic curves. The rigorous determination of these IB cutoff values allowed tissue characterization of plaque components with high diagnostic accuracy. In this study, there was excellent agreement between the IB-IVUS and histological findings (Cohen's $\kappa$-value of 0.83 ).

\section{Thickness of the Fibrous Cap}

It was reported recently that OCT is a reliable and reproducible imaging modality for tissue characterization. Atherosclerotic plaques can be discriminated ex vivo with a high degree of sensitivity (90-94\%) and specificity (90-92\%). ${ }^{3}$ OCT imaging enables clear cross-sectional imaging at discrete locations in vivo, although this method requires removal of blood from the coronary arteries in the field of the view to obtain a high-quality image, and the limited penetration depth precludes comprehensive evaluation of the entire vessel. Kume et al reported that there was good correlation of the thickness of the fibrous cap between OCT and histological examination $(\mathrm{y}=0.97 \mathrm{x}+28.49 ; \mathrm{r}=0.90 ; \mathrm{P}<0.001) .{ }^{10}$ The mean difference in the thickness of the fibrous cap measured by OCT and histological examination was $-24 \pm 44 \mu \mathrm{m}$ in that study.

Although OCT has been used to evaluate the thickness of fibrous caps, evaluation of human coronary plaques using 
IVUS has not been performed. The present study demonstrated that the thickness of the fibrous cap measured by IB-IVUS with improved resolution correlated with that measured by OCT. However, the strength of the correlation $(r=$ $0.74)$ and the standard deviation of mean difference $(-2 \pm$ $147 \mu \mathrm{m}$ ) between IB-IVUS and OCT was not as good as that previously reported between OCT and histology $(r=0.90$ and $-24 \pm 44 \mu \mathrm{m}$ ), because of the relatively long wavelength of the ultrasound signal compared with light.

A pathological study demonstrated that coronary stenting accompanied by penetration of the stent into the lipid core induces increased arterial inflammation, which is associated with restenosis after stenting. ${ }^{22}$ That finding suggested the importance of evaluating the thickness of the fibrous cap overlaying the lipid core to avoid chronic restenosis after coronary stenting. In a previous virtual histology IVUS study, thincap fibroatheroma (TCFA) was provisionally defined as $>3$ consecutive frames (percent atheroma $\geq 40 \%$ ) consisting of necrotic core $\geq 10 \%$ without evident overlying fibrous tissue, ${ }^{23}$ but there was no comparative study of the thickness of the fibrous cap measured by virtual histology IVUS and OCT. Ours is the first study to use IB-IVUS to measure the thickness of the fibrous cap.

\section{Study Limitations}

There were a few limitations of the present method. First, the angle dependence of the ultrasound signal makes tissue characterization unstable when lesions are not perpendicular to the axis. Picano et al reported that angular scattering behavior is large in calcified and fibrous tissues, whereas it is slight to null in normal and fatty plaque. ${ }^{24}$ According to that report, although there was no crossover of IB values between fibrous and fibrofatty tissue within an angle span of $10^{\circ}$, or between fibrous and fatty tissue within the angle span of $14^{\circ}$, the angle dependency of the ultrasound signal might be partially responsible for the variation of the IB values obtained from each tissue component. There is a report that demonstrated in detail that the angle dependence of $30-\mathrm{MHz}$ ultrasound in the arterial intima and media is $1.11 \mathrm{~dB} / 10^{\circ} .{ }^{25}$ When we used a $40-\mathrm{MHz}$ catheter, the angle dependence increased in arterial tissue and this angle dependence of the ultrasound signal may decrease its accuracy in differentiating tissue components.

Second, calcification is a perfect reflector for ultrasound, causing the acoustic shadowing so typical in the IVUS images. The ultrasound signals cannot penetrate, or pass through, the calcified layer and are reflected back towards the transducer. ${ }^{26}$ Therefore, accurate tissue characterization of the areas behind calcification is not possible as with conventional IVUS and may also decrease the diagnostic accuracy.

Third, the pulsation of the coronary arteries hindered rigorous comparison of the IB-IVUS and OCT images, because the comparison was performed in vivo. The shape of the vessel lumen was slightly different in the IB-IVUS and OCT images, even in the same cross-sections, because it was difficult to obtain the 2 images during the same cardiac cycle. The shape of the luminal surface may affect the imaging of the fibrous cap by IB-IVUS and OCT.

Finally, the minimum thickness of the fibrous cap included in the present study was $80 \mu \mathrm{m}$. IB-IVUS cannot discriminate TCFA consisting of a thin fibrous cap $(\leq 65 \mu \mathrm{m})$ because of the limited resolution of current ultrasound imaging technology. However, Nissen ${ }^{27}$ has recently criticized an arbitrary attempt to define a continuous phenomenon as a series of discrete variables in which a plaque with cap thickness of
$66 \mu \mathrm{m}$ would not be a TCFA, but a plaque with cap thickness of $64 \mu \mathrm{m}$ would be a high-risk lesion. He also criticized the assumption by most investigators in this field that there is a direct 1-to-1 relationship between a TCFA and a high-risk plaque based on the limited autopsy studies of Virmani et al. ${ }^{28}$ In addition, a recent OCT study reported that the fibrous cap could rupture during exercise, even when its thickness was $>65 \mu \mathrm{m} .{ }^{6}$ Further studies that systematically evaluate the continuous plaque phenomenon are needed to understand the relationship between fibrous cap thickness and plaque vulnerability in the clinical setting.

\section{Conclusions}

We improved an online IB-IVUS imaging system for tissue characterization of coronary plaques. The use of the appropriate IB cutoff points for discriminating lipid pool, fibrosis and calcification resulted in tissue characterization with a high diagnostic accuracy. The thickness of the fibrous cap measured by IB-IVUS correlated with that measured by OCT.

\section{Acknowledgments}

This study was supported, in part, by a research grant of (20590822, 2009) from the Ministry of Education, Culture, Sports, Science and Technology of Japan.

\section{Disclosure}

We have no financial or other relations that could lead to conflict of interest.

\section{References}

1. Horie T, Sekiguchi M, Hirosawa K. Coronary thrombosis in pathogenesis of acute myocardial infarction: Histopathological study of coronary arteries in 108 necropsied cases using serial section. $\mathrm{Br}$ Heart J 1978; 40: 153-161.

2. Mizuno K, Satomura K, Miyamoto A, Arakawa K, Shibuya T, Arai T, et al. Angioscopic evaluation of coronary artery thrombi in acute coronary syndromes. N Engl J Med 1992; 326: 287-291.

3. Yabushita H, Bouma BE, Houser SL, Aretz HT, Jang IK, Schlendorf $\mathrm{KH}$, et al. Characterization of human atherosclerosis by optical coherence tomography. Circulation 2002; 106: 1640-1645.

4. Jang IK, Bouma BE, Kang DH, Park SJ, Park SW, Seung KB, et al. Visualization of coronary atherosclerotic plaques in patients using optical coherence tomography: Comparison with intravascular ultrasound. J Am Coll Cardiol 2002; 39: 604-609.

5. Jang IK, Tearney GJ, MacNeill B, Takano M, Moselewski F, Iftima $\mathrm{N}$, et al. In vivo characterization of coronary atherosclerotic plaque by use of optical coherence tomography. Circulation 2005; 111: $1551-1555$.

6. Tanaka A, Imanishi T, Kitabata H, Kubo T, Takarada S, Tanimoto $\mathrm{T}$, et al. Morphology of exertion-triggered plaque rupture in patients with acute coronary syndrome: An optical coherence tomography study. Circulation 2008; 118: 2368-2373.

7. Kume T, Okura H, Yamada R, Kawamoto T, Watanabe N, Neishi $\mathrm{Y}$, et al. Frequency and spatial distribution of thin-cap fibroatheroma assessed by 3-vessel intravascular ultrasound and optical coherence tomography: An ex vivo validation and an initial in vivo feasibility study. Circ J 2009; 73: 1086-1091.

8. Sawada T, Shite J, Negi N, Shinke T, Tanino Y, Ogasawara D, et al. Factors that influence measurements and accurate evaluation of stent apposition by optical coherence tomography: Assessment using a phantom model. Circ J 2009; 73: 1841-1847.

9. Kawasaki M, Bouma BE, Bressner J, Houser SL, Nadkarni SK, MacNeill BD, et al. Diagnostic accuracy of optical coherence tomography and integrated backscatter intravascular ultrasound images for tissue characterization of human coronary plaques. J Am Coll Cardiol 2006; 48: 81-88.

10. Kume T, Akasaka T, Kawamoto T, Okura H, Watanabe N, Toyota $\mathrm{E}$, et al. Measurement of the thickness of the fibrous cap by optical coherence tomography. Am Heart J 2006; 152: 755.e1 -e4.

11. Kawasaki M, Takatsu H, Noda T, Ito Y, Kunishima A, Arai M, et al. Non-invasive tissue characterization of human atherosclerotic 
lesions in carotid and femoral arteries by ultrasound integrated backscatter: Comparison between histology and integrated backscatter images before and after death. J Am Coll Cardiol 2001; 38: 486-492.

12. Kawasaki M, Takatsu H, Noda T, Sano K, Ito Y, Hayakawa K, et al. In vivo quantitative tissue characterization of human coronary arterial plaques by use of integrated backscatter intravascular ultrasound and comparison with angioscopic findings. Circulation 2002; 105: $2487-2492$.

13. Okubo M, Kawasaki M, Ishihara Y, Takeyama U, Yasuda S, Kubota $\mathrm{T}$, et al. Tissue characterization of coronary plaques: Comparison of integrated backscatter intravascular ultrasound with virtual histology intravascular ultrasound. Circ J 2008; 72: 1631-1639.

14. Sano K, Kawasaki M, Okubo M, Yokoyama H, Ito Y, Murata I, et al. In vivo quantitative tissue characterization of angiographically normal coronary lesions and the relation with risk factors: A study using integrated backscatter intravascular ultrasound. Circ J 2005; 69: $543-549$

15. Sano K, Kawasaki M, Ishihara Y, Okubo M, Tsuchiya K, Nishigaki $\mathrm{K}$, et al. Assessment of vulnerable plaques causing acute coronary syndrome using integrated backscatter intravascular ultrasound. J Am Coll Cardiol 2006; 47: 734-741.

16. Shung KK, Reid JM. The acoustical properties of deoxygenated sickle cell blood and hemoglobin S solution. Ann Biomed Eng 1977; 5: $150-156$.

17. Okubo M, Kawasaki M, Ishihara Y, Takeyama U, Kubota T, Yamaki T, et al. Development of integrated backscatter intravascular ultrasound for tissue characterization of coronary plaques. Ultrasound Med Biol 2008; 34: 655-663.

18. Prati F, Arbustini E, Labellarte A, Dal Bello B, Sommariva L, Mallus MT, et al. Correlation between high frequency intravascular ultrasound and histomorphology in human coronary arteries. Heart 2001; 85: 567-570.

19. Cohen J. A coefficient of agreement for nominal scales. Educ Psy- chol Measure 1960; 20: 37-46.

20. Fleiss JL. Statistical methods for rates and proportions, 2nd edn. New York: John Wiley \& Son, 1981.

21. Nasu K, Tsuchikane E, Katoh O, Vince DG, Virmani R, Surmely $\mathrm{JF}$, et al. Accuracy of in vivo coronary plaque morphology assessment: A validation study of in vivo virtual histology compared with in vitro histopathology. J Am Coll Cardiol 2006; 47: 2405-2412.

22. Farb A, Weber DK, Kolodgie FD, Burke AP, Virmani R. Morphological predictors of restenosis after coronary stenting in humans. Circulation 2002; 105: 2974-2980.

23. Rodriguez-Granillo GA, García-García HM, Mc Fadden EP, Valgimigli M, Aoki J, de Feyter P, et al. In vivo intravascular ultrasound-derived thin-cap fibroatheroma detection using ultrasound radiofrequency data analysis. J Am Coll Cardiol 2005; 46: $2038-2042$.

24. Picano E, Landini L, Distante A, Salvadori M, Lattanzi F, Masini $\mathrm{M}$, et al. Angle dependence of ultrasonic backscatter in arterial tissues: A study in vitro. Circulation 1985; 72: 572-576.

25. Courtney BK, Robertson AL, Maehara A, Luna J, Kitamura K, Morino Y, et al. Effect of transducer position on backscattered intensity in coronary arteries. Ultrasound Med Biol 2002; 28: 81-91.

26. Mintz GS, Nissen SE, Anderson WD, Bailey SR, Erbel R, Fitzgerald PJ, et al. American College of Cardiology clinical expert consensus document on standards for acquisition, measurement and reporting of intravascular ultrasound studies (IVUS): A report of the American College of Cardiology task force on clinical expert consensus documents developed in collaboration with the European society of cardiology endorsed by the society of cardiac angiography and interventions. J Am Coll Cardiol 2001; 37: 1478-1492.

27. Nissen SE. The vulnerable plaque "hypothesis": Promise, but little progress. JACC Cardiovasc Imaging 2009; 2: 483-485.

28. Virmani R, Burke AP, Farb A, Kolodgie FD. Pathology of the vulnerable plaque. J Am Coll Cardiol 2006; 47(Suppl): C13-C18. 